Discussion Papers in

Economics and Econometrics

2000

This paper is available on our website http://www/soton.ac.uk/ econweb/dp/dp00.html 


\title{
A simple locally interactive model of ergodic and nonergodic growth*
}

\author{
Valentina Corradi \\ Department of Economics \\ Queen Mary and Westfield College \\ Mile End Road \\ London E1 4NS, UK \\ V.Corradi@qmw.ac.uk \\ Antonella Ianni \\ Department of Economics \\ University of Southampton \\ Highfield \\ Southampton, SO17 1BJ, UK \\ A.Ianni@soton.ac.uk
}

April 2000

Keywords: clusters, contagion, local interaction, nonergodic growth, nonlinear voter model.

\footnotetext{
${ }^{*}$ We wish to thank Luca Anderlini, David Cass, Giulio Fella, Christian Ghiglino, Paul Labys, Larry Samuelson, Gerhard Sorger and the seminar participants at the University of Exeter for helpful comments and suggestions on previous versions of this paper.
} 


\begin{abstract}
In this paper we propose a locally interactive model which explains both the cross sectional dynamics as well as the possibility of multiple long run equilibria. Firms can choose between two technologies say 1 and 0 ; the returns from technology 1 are affected by the number of neighboring firms using it; the returns from technology 0 are independent of neighboring firms technological choices. Durlauf (1993) explains nonergodic growth via strong technological complementarities. By modeling in a different way the transmission of the spillover effects, we show that in presence of technological complementarities of intermediate strength we have either two or infinitely many long run equilibria. The basin of attraction of these equilibria depend on the initial conditions. On the other hand when the technological complementarities are either very weak or very strong then we have a unique long run equilibrium. As for the dynamic behavior, we shall explain the formation of large connected areas, clusters. As the cluster size grows at a rate slower than $t$, such areas seem to be stationary along the dynamics.
\end{abstract}




\section{Introduction}

According to the standard one-sector model (e.g. Solow (1956)) economies sharing the same technology and the same preferences should tend to converge to the same balanced growth path, no matter what are their initial conditions. However this convergence prediction is violated by the empirical evidence. For example Durlauf and Quah (1999) point out that, despite the stability of the population size distribution, the cross country distribution of income seems quite volatile. In particular the poor seem to become poorer and the rich to become richer. Broadly speaking, if countries with identical technology and preferences tend to reach a common path of balanced growth, no matter what are their initial conditions, we should believe that growth is an ergodic process. On the other hand if countries starting from different conditions tend to reach different growth paths, we may think that instead growth is a nonergodic process. Over the last couple of decades there have been proposed several explanations for the observed divergence and for the existence of multiple steady states. One approach explains the observed divergence in growth via human (or physical) capital accumulation by individuals, which is responsible for spillover effects that, if strong enough, lead to multiple equilibria. For example Azariadis and Drazen (1990) consider the case of threshold externalities, so that countries that start from a level of capital below a given threshold converge to a low steady state, while countries that start with a level of capital above a given threshold converge to a high steady state. Other explanations for multiple equilibria are the joint presence of increasing returns together with market imperfections (e.g. Murphy, Shleifer and Vishny 1989) and the joint presence of strategic interaction together with coordination failure (e.g. Cooper and John, 1988). Nevertheless the literature cited above mainly explains the possibility of multiple steady state equilibria, but it does not focus on the dynamics, so that it cannot explain the cyclical behavior of the aggregate output or the often observed cross sectional heterogeneity around a given equilibrium.

In order to study cycles in aggregate activity as well as cross sectional heterogeneity around a given steady state, in a seminal paper Durlauf (1993) relies on a spatial-temporal analysis based on random fields theory. In Durlauf's (1993) growth model there are two available technologies and one of the two is more efficient than the other. More precisely the efficiency advantage increases the higher is the number of neighboring firms using that technology. His locally interactive model can explain both output volatility across firms as well as multiple long run equilibria. The strength 
of complementarities is measured by the probability that a firm uses the high technology at time $t$, given that some neighboring firms were using the low technology at time $t-1$. A low value of such probability indicates powerful complementarities, while a high value indicates weak complementarities. If such probability is above a given threshold, then an economy starting from a low equilibrium (all firms using the low technology) almost surely will converge to the high equilibrium (all firms using the high technology). As a consequence, the average output level of each industry is the same regardless of whether the economy starts from a low or a high initial state. On the other hand, if such probability is below a given threshold, then with probability one an economy starting from a low initial state will not converge to the high equilibrium. Thus nonergodic growth and multiple equilibria can arise as a consequence of strong complementarities and spillover effects. ${ }^{1}$

In this paper we propose a locally interactive model which explains both the cross sectional dynamics as well as the possibility of multiple long run equilibria. In our model there is only one factor, capital, which becomes obsolete at a random Poisson time, with intensity parameter equal to one, so that the average life of capital is equal to one. Each time capital becomes obsolete, the firm can reconsider its technology choice. There are indeed two technologies, one, say technology 1 is more (less) efficient than the other, say technology 0 , when the number of neighbors using 1 is above (below) a certain threshold. Thus any time capital becomes obsolete the firm (there is a countable number of firms living on a one dimensional lattice) chooses either technology 0 or 1 depending on whether the numbers of neighbors using 1 is above or below a given threshold. Either a configuration with all firms using technology 0 or a configuration with all firms using technology 1 are absorbing states for our model. We show that in the case of very strong or very weak complementarities, we have a unique long run equilibrium, starting from any non degenerate initial distribution. More precisely in the case of strong technological complementarities the unique long run equilibrium will be a configuration of all 1 , while in the case of weak technological complementarities the only long run equilibrium will be a configuration of all $0^{2}$. On the other hand, multiple equilibria and so

\footnotetext{
${ }^{1}$ Models of local interaction and spillovers from neighbors as an explanation for multiple equilibria in human capital accumulation or ghettos formations have also been proposed (see e.g. Durlauf 1994, Brock and Durlauf (2000) and references therein). For example Bala and Sorger (1999) show that local spillovers affect the return from investment in education, leading to multiple equilibria characterized by different social stratification in terms of workers and managers. A local interactive model that explains formation and persistence of ghettos (consistent with the history of US black ghettos) is recently proposed by Möbius (1999).

${ }^{2}$ This in contrast with Durlauf's model, in which when tecnological complementarities are weak we have conver-
} 
nonergodicity, arise for intermediate strength of complementarities. In fact in the intermediate case different outcomes may occur in the long run depending on the behavioral rule chosen by the firm when half of the neighbors (the number of neighbors is supposed to be even) are using technology one. If any time half of neighbors are using technology 1 (and so half are using technology 0 ), the firm either switches technology or flips a coin and chooses 0 or 1 with probability $1 / 2$, then there are two possible equilibria, all 0 or all 1 . Also their basins of attraction depend on the number of firms using 1 in the initial state. Broadly speaking, the equilibrium with all 1 (say the high equilibrium) is more likely to occur, the higher is the number of firms using 1 in the initial state. Finally, if whenever half of the neighbors use technology one, the firm keeps the technology currently used, then we shall have countably many long run equilibria, i.e. infinitely many technological configurations can survive in the long run, including the two degenerate equilibria. In this sense we infer that while technological spillovers are a main explanation for nonergodic growth, the fact that either a very strong or a very weak or an intermediate degree of technological complementarities leads to multiple steady states depends on the specific modeling of the spillover transmission. As for what concerns the dynamic behavior, we shall explain the formation of large homogeneous areas, the so called clusters. As the cluster size grows at a rate slower than $t$, such areas seem to be stationary along the dynamics. Thus clusters may provide an explanation to the stylized fact that large wealthy geographical areas are alternated with large depressed areas.

The paper is organized as follows. Section 2 describes the model. Section 3 describes the aggregate process of technology choice in the case of complementarities of intermediate strength, that is when the threshold parameter is equal to $1 / 2$ (symmetric case). In particular we show that the process of technological choice can be seen as either a linear or a nonlinear voter model. Relying on some of the results available in the probability literature for the linear and the nonlinear voter model, Section 4 describes the asymptotics and the dynamics of the aggregate process in the symmetric case. Section 5 describe the asymptotics and the dynamic behavior of the aggregate technology process in the asymmetric case, that is when to flip from technology 0 (1) to technology 1 (0) it requires a smaller (larger) number of neighboring firms disagreeing on the technology choice. Some concluding remarks are given in Section 6.

gence to a configuration of all 1 . This is mainly due to the fact that in our model both the degenerate configurations (all 0 or all 1 ) are absorbing states, while in Durlauf only a configuration with all 1 is an absorbing state. 


\section{The Model}

There is a countable number of identical firms, living on a one dimensional lattice, Z. Each firm is characterized by its location on the lattice, that is firm $x$ is located at $x \in Z$. Firms cannot change locations. Each of them produces a homogeneous good, which may be used either as capital or paid out as dividends. Firms can choose between two different technologies, say Technology 0 and Technology 1 , both of them are strictly concave in capital. The aggregate output is determined by local interaction among different firms. In particular the choice of the technology is affected by the number of neighboring firms adopting that technology. The returns from technology 0 are independent of the number of neighboring firms using it, while the return from technology 1 receive a positive (negative) spillover if the number of neighbors using it is above (below) a given threshold.

At time 0 , each firm receives an identical endowment of capital, technology are randomly (and independently) assigned to firms. Given the initial endowment of capital, production occurs in continuous time. However capital gets obsolete at a random Poisson time with intensity parameter equal to one, i.e. the expected life is one. The random times at which capital becomes obsolete are independent across firms. When capital becomes obsolete the firm looks at the neighboring firms and, depending whether the fraction of neighbors using technology 1 is above or below a certain threshold, firm $x$ chooses technology 1 or 0 , and begin to produce the homogeneous good until capital gets obsolete again. Because of the Poisson assumption, the probability that more than one firm reconsider its technology in the same instant of time is zero.

\subsection{Notation and Set up}

$x=\ldots-m,-m+1, \ldots-1,0,1, \ldots m-1, m, \ldots$ denotes the location of the firm on the lattice, and so $x \in Z$.

$l=0,1$ denotes the technology chosen.

$\tau_{i}^{x}$ denotes the random time at which firm $x$ reconsider the technology chosen for the $i-t h$ time. Thus $\tau_{i+1}^{x}-\tau_{i}^{x}$ denotes the (random) time elapsed between the $i-t h$ and the $i+1-t h$ time firm $x$ can reconsider its production technology.

$K_{x}\left(\tau_{i}^{x}\right)$ is the stock of capital of firm $x$ at time $\tau_{i}^{x} . Y_{x, l}(t)$ denotes the cumulative output (from $\tau_{i}^{x}$ to $t$ ) of firm $x$ when using technology $l$. For any $i=1,2, \ldots$ at time $\tau_{i}^{x}$ the capital of firm $x$ becomes obsolete and the firm has the opportunity of reconsidering the technology choice. For 
$\tau_{i}^{x} \leq t<\tau_{i+1}^{x}$, if firm $x$ employs technology 0,

$$
d Y_{x, \mathbf{0}}(t)=f_{0}\left(K_{x}\left(\tau_{i}^{x}\right), \sum_{h=x-N / 2, h \neq x}^{x+N / 2} \eta_{h}\left(\tau_{i}^{x}\right)\right) d t
$$

otherwise if firm $x$ employs technology 1 ,

$$
d Y_{x, 1}(t)=f_{1}\left(K_{x}\left(\tau_{i}^{x}\right), \sum_{h=x-N / 2, h \neq x}^{x+N / 2} \eta_{h}\left(\tau_{i}^{x}\right)\right) d t
$$

where $\eta_{h}\left(\tau_{i}^{x}\right)=1$ if the firm located at site $h$ at time $\tau_{i}^{x}$ is employing technology 1 , and $\eta_{h}\left(\tau_{i}^{x}\right)=0$ if instead firm $h$ at time $\tau_{i}^{x}$ is employing technology 0.

For any realization of $\tau_{i}^{x}$ and for all possible realizations, firm $x$ solves the following profit maximization problem,

$$
\begin{aligned}
& \max _{K_{x}\left(\tau_{i}^{x}\right)} E\left[\operatorname { m a x } \left\{\int_{\tau_{i}^{x}}^{\tau_{i+1}^{x}} f_{0}\left(K_{x}\left(\tau_{i}^{x}\right), \sum_{h=x-N / 2, h \neq j}^{x+N / 2} \eta_{h}\left(\tau_{i}^{x}\right)\right) d t-K_{x}\left(\tau_{i}^{x}\right),\right.\right. \\
& \left.\left.\int_{\tau_{i}^{x}}^{\tau_{i+1}^{x}} f_{1}\left(K_{x}\left(\tau_{i}^{x}\right), \sum_{h=x-N / 2, h \neq x}^{x+N / 2} \eta_{h}\left(\tau_{i}^{x}\right)\right) d t-K_{x}\left(\tau_{i}^{x}\right)\right\}\right]
\end{aligned}
$$

Note that conditional on a realization of $\tau_{i}^{x}$ (and for all realization), the only random element in (1) is $\tau_{i+1}^{x}$, that is the time at which the new stock of capital will become obsolete.

\subsection{Assumptions}

\section{A1-Random Times}

$\forall i, x \tau_{i+1}^{x}-\tau_{i}^{x}$ are identically and independently distributed, with $\operatorname{Pr}\left(\tau_{i+1}^{x}-\tau_{i}^{x}>z\right)=\exp (-z)$, so that $E\left(\tau_{i+1}^{x}-\tau_{i}^{x}\right)=1$. Also $\tau_{i}^{x}$ and $\tau_{m}^{n}$ are independent $\forall i \neq m$ and/or $x \neq n$.

A1 ensures that at any instant of time the probability that more than one firm reconsider its technology choice is zero, also at any instant, each firm has a positive probability of reconsidering its technology. It also ensures that the average life of capital is equal to one. As $\tau_{i}^{x}$ and $\tau_{m}^{n}$ are independent $\forall i \neq m$ and/or $x \neq n$, the random life of capital plays the same role of the $i i d$ random shocks in Durlauf's model.

\section{A2-Technology}

$\forall x \in Z, l=0,1$, 
(i) $f_{l}\left(0, \sum_{h=x-N / 2, h \neq x}^{x+N / 2} \eta_{h}\left(\tau_{i}^{x}\right)\right)=0$

(ii) $\partial f_{l}\left(0, \sum_{h=x-N / 2, h \neq x}^{x+N / 2} \eta_{h}\left(\tau_{i}^{x}\right)\right) / \partial k=\infty$ and $\partial f_{l}\left(\infty, \sum_{h=x-N / 2, h \neq x}^{x+N / 2} \eta_{h}\left(\tau_{i}^{x}\right)\right) / \partial k=0$.

(iii) $\partial f_{l} / \partial K>0, \partial^{2} f_{l} / \partial K^{2}<0$.

A2 is a standard assumption ruling out convexity in production. This ensures the existence of a unique optimal stock of capital, each time the firm faces the maximization problem in (1).

\section{A3-Technology Spillovers}

For any realization of $\tau_{i}^{x}, \forall i, x$ and for any level of capital stock $K_{x}\left(\tau_{i}^{x}\right)$,

(i) $f_{0}\left(K_{x}\left(\tau_{i}^{x}\right), \sum_{h=x-N / 2, h \neq x}^{x+N / 2} \eta_{h}\left(\tau_{i}^{x}\right)\right)=f_{0}\left(K_{x}\left(\tau_{i}^{x}\right)\right)$

Thus the returns from technology 0 are not affected by the neighboring firms' decisions.

Hereafter $\delta \in(0,1)$ and is such that $\delta N$ is an integer, where $N$ is assumed to be an even number. We now describe how local interaction affects the output growth.

If $\sum_{h=x-N / 2, h \neq x}^{x=N / 2} \eta_{h}\left(\tau_{i}^{x}\right)<\delta N$, then $f_{0}\left(K_{x}\left(\tau_{i}^{x}\right)\right)>f_{1}\left(K_{x}\left(\tau_{i}^{x}\right), \sum_{h=x-N / 2, h \neq x}^{x+N / 2} \eta_{h}\left(\tau_{i}^{x}\right)\right)$, if instead $\sum_{h=x-N / 2, h \neq x}^{x+N / 2} \eta_{h}\left(\tau_{i}^{x}\right)>\delta N$, then $f_{0}\left(K_{x}\left(\tau_{i}^{x}\right)\right)<f_{1}\left(K_{x}\left(\tau_{i}^{x}\right), \sum_{h=x-N / 2, h \neq x}^{x+N / 2} \eta_{h}\left(\tau_{i}^{x}\right)\right)$ and if $\sum_{h=x-N / 2, h \neq x}^{x+N / 2} \eta_{h}\left(\tau_{i}^{x}\right)=\delta N$, then $f_{0}\left(K_{x}\left(\tau_{i}^{x}\right)\right)=f_{1}\left(K_{x}\left(\tau_{i}^{x}\right), \sum_{h=x-N / 2, h \neq x}^{x+N / 2} \eta_{h}\left(\tau_{i}^{x}\right)\right)$

(iii) For any integer $m \neq n$ and either $m, n>\delta N$ or $m, n<\delta N$,

$$
f_{1}\left(K_{x}\left(\tau_{i}^{x}\right), \sum_{h=x-N / 2, h \neq x}^{x+N / 2} \eta_{h}\left(\tau_{i}^{x}\right)=m\right)=f_{1}\left(K_{x}\left(\tau_{i}^{x}\right), \sum_{h=x-N / 2, h \neq x}^{x+N / 2} \eta_{h}\left(\tau_{i}^{x}\right)=n\right)
$$

The returns from technology 1 receive a positive (negative) externality, depending on whether the fraction of neighbors using it is below or above a certain threshold. Note that the externality effects depend on the fraction of neighbors using technology 1 , but not necessarily on whether the firm was previously using technology 1 or 0 . In this sense the positive effect due to a large fraction of identical neighbors using technology 1, cannot be interpreted as a learning by doing effect. We shall just interpret them as generic technological spillovers and complementarities. Finally (iii) ensures that the intensity of the spillover effects just depends on whether the number of neighbors using technology 1 is above or below a given threshold, and not on the actual number of neighbors using 1.

\section{A4-Initial conditions}

At time 0 each firm receives an identical endowment of capital, say $K_{x}(0)=K(0), \forall x$. Then each firm takes an independent draw from a uniform $[0,1]$ random variable, if the realization of 
firm $x$ draw is equal to or smaller than $\theta, \theta \in(0,1)$, then firm $x$ chooses technology 1 , if instead firm $x$ draws a realization larger than $\theta$, then it uses technology 0 .

A4 rule out the possibility of a degenerate initial state. It also ensures that the probability measure governing the initial configuration of technologies is a Bernoulli product measure, and so it is translation invariant, i.e. independent of the specific site.

An example of technologies satisfying A2 and A3 are, for $\tau_{i}^{x} \leq t<\tau_{i+1}^{x}$,

$$
d Y_{x, 0}(t)=A K_{x}\left(\tau_{i}^{x}\right)^{\alpha} d t
$$

and

$$
d Y_{x, 1}(t)=\left(A+1\left\{\sum_{h=x-N / 2, h \neq x}^{x+N / 2} \eta_{h}\left(\tau_{1}^{x}\right)>\delta N\right\}-1\left\{\sum_{h=x-N / 2, h \neq x}^{x+N / 2} \eta_{h}\left(\tau_{1}^{x}\right)<\delta N\right\}\right) K_{x}\left(\tau_{i}^{x}\right)^{\alpha} d t
$$

with $0<\alpha<1,1<A<\infty$.

\subsection{Heuristics}

At time 0 firm $x$ receives its initial endowment of capital, say $K_{x}(0)=K(0) \forall x$, with probability $\theta$ (resp. $(1-\theta)$ ) firm $x$ begins to produce with technology 1 (resp. 0). Note that for $\theta=1$ (resp. $\theta=0$ ) a configuration with all firms using technology 1 (resp.0) is an absorbing state. For this reason we impose (A4) $\theta \in(0,1)$. At (random) times $\tau_{i}^{x} i=1,2, \ldots$ the initial stock of capital becomes obsolete and so firm $x$ can reconsider its choice of technology. Conditional on the realization of $\tau_{i}^{x}$, and for all realizations but a set of measure zero, and on the technology adopted by the $N$ neighbors at this time, we have that if $\sum_{h=x-N / 2, h \neq x}^{x+N / 2} \eta_{h}\left(\tau_{i}^{x}\right)>$ $\delta N$ then $f_{0}\left(K_{x}\left(\tau_{i}^{x}\right)\right)<f_{1}\left(K_{x}\left(\tau_{i}^{x}\right), \sum_{h=x-N / 2, h \neq x}^{x+N / 2} \eta_{h}\left(\tau_{i}^{x}\right)\right)$, while if $\sum_{h=x-N / 2, h \neq x}^{x+N / 2} \eta_{h}\left(\tau_{i}^{x}\right)<\delta N$ then $f_{0}\left(K_{x}\left(\tau_{i}^{x}\right)\right)>f_{1}\left(K_{x}\left(\tau_{i}^{x}\right), \sum_{h=x-N / 2, h \neq j}^{x+N / 2} \eta_{h}\left(\tau_{i}^{x}\right)\right)$. Finally, if $\sum_{h=x-N / 2, h \neq x}^{x+N / 2} \eta_{h}\left(\tau_{i}^{x}\right)=\delta N$ then $f_{0}\left(K_{x}\left(\tau_{i}^{x}\right)\right)=f_{1}\left(K_{x}\left(\tau_{1}^{x}\right) \sum_{h=x-N / 2, h \neq j}^{x+N / 2} \eta_{h}\left(\tau_{1}^{x}\right)\right)$. Suppose that $\sum_{h=x-N / 2, h \neq x}^{x+N / 2} \eta_{h}\left(\tau_{i}^{x}\right)>\delta N$, then the profit maximization problem for firm $x$ is

$$
\max _{K_{x}\left(\tau_{i}^{x}\right)} E\left[\int_{\tau_{i}^{x}}^{\tau_{i+1}^{x}} f_{1}\left(K_{x}\left(\tau_{i}^{x}\right), \sum_{h=x-N / 2, h \neq x}^{x+N / 2} \eta_{h}\left(\tau_{i}^{x}\right)\right) d t-K_{x}\left(\tau_{i}^{x}\right)\right]
$$

For any given realization of $\tau_{i}^{x}$ and for the neighbors technology choices, the only random element in the maximization problem above is $\tau_{i+1}^{x}$, that is the time in which the new capital stock 
will become obsolete. However given $A 1$ we have that $E\left(\tau_{i+1}^{x}-\tau_{i}^{x}\right)=1, \forall i$, so that the profit maximization above can be rewritten as

$$
\begin{aligned}
& \max _{K_{x}\left(\tau_{1}^{x}\right)}\left(f_{1}\left(K_{x}\left(\tau_{i}^{x}\right), \sum_{h=x-N / 2, h \neq x}^{x+N / 2} \eta_{h}\left(\tau_{i}^{x}\right)\right) E\left[\int_{\tau_{i}^{x}}^{\tau_{i+1}^{x}} d t\right]-K_{x}\left(\tau_{i}^{x}\right)\right) \\
= & \max _{K_{x}\left(\tau_{1}^{x}\right)}\left(f_{1}\left(K_{x}\left(\tau_{i}^{x}\right), \sum_{h=x-N / 2, h \neq x}^{x+N / 2} \eta_{h}\left(\tau_{i}^{x}\right)\right)-K_{x}\left(\tau_{i}^{x}\right)\right)
\end{aligned}
$$

¿From (4) we see that the optimal level of capital is given by the solution to

$$
f_{1}^{\prime}\left(K_{x}\left(\tau_{i}^{x}\right), \sum_{h=x-N / 2, h \neq x}^{x+N / 2} \eta_{h}\left(\tau_{i}^{x}\right)\right)=1
$$

so that $K_{x}^{*}\left(\tau_{i}^{x}\right)=k_{1}^{*} \forall i, x$. On the other hand whenever $\sum_{h=x-N / 2, h \neq x}^{x+N / 2} \eta_{h}\left(\tau_{i}^{x}\right)<\delta N$ the firm chooses technology 0 and the profit maximization problem becomes ${ }^{3}$

$$
\max _{K_{x}\left(\tau_{i}^{x}\right)} E\left[\int_{\tau_{i}^{x}}^{\tau_{i+1}^{x}} f_{0}\left(K_{x}\left(\tau_{i}^{x}\right), \sum_{h=x-N / 2, h \neq x}^{x+N / 2} \eta_{h}\left(\tau_{i}^{x}\right)\right) d t-K_{x}\left(\tau_{i}^{x}\right)\right]
$$

so the optimal level of capital is given by the solution to

$$
f_{0}^{\prime}\left(K_{x}\left(\tau_{i}^{x}\right), \sum_{h=x-N / 2, h \neq x}^{x+N / 2} \eta_{h}\left(\tau_{i}^{x}\right)\right)=1
$$

that is $K_{x}^{*}\left(\tau_{i}^{x}\right)=k_{0}^{*} \forall i, x$. So the optimal stock of capital can takes only two values, $k_{0}^{*}$, when technology 0 is employed and $k_{1}^{*}$ when technology 1 is employed, where $k_{0}^{*}<k_{1}^{*}$. In terms of our example (2)(3) it is immediate to see that $k_{1}^{*}=(2 \alpha A)^{\frac{1}{1-\alpha}}$, and $k_{0}^{*}=(\alpha A)^{\frac{1}{1-\alpha}}$. Thus $\forall x, i$ and for $\tau_{i}^{x} \leq t<\tau_{i+1}^{x}$, recalling $\mathrm{A} 2$ and $\mathrm{A} 3$ the output grows in continuous time according to either

$$
d Y_{x, 0}(t)=f_{0}\left(k_{\mathbf{0}}^{*}, \sum_{h=x-N / 2, h \neq x}^{x+N / 2} \eta_{h}\left(\tau_{i}^{x}\right)\right) d t=\bar{f}_{\mathbf{0}} d t
$$

or

$$
d Y_{x, 1}(t)=f_{1}\left(k_{1}^{*}, \sum_{h=x-N / 2, h \neq x}^{x+N / 2} \eta_{h}\left(\tau_{i}^{x}\right)\right) d t=\bar{f}_{1} d t
$$

where $\bar{f}_{0}<\bar{f}_{1} \cdot{ }^{4}$ Thus along the capital life, the rate of growth of output is constant and is completely determined by the technology choice.

\footnotetext{
${ }^{3}$ The behavior of the firm when $\sum_{h=x-N / 2, h \neq x}^{x+N / 2} \eta_{h}\left(\tau_{i}^{x}\right)=\delta N$ will be analyzed in detail in sections 3 and 5 .

${ }^{4}$ Note that whenever $\sum_{h=x-N / 2, h \neq x}^{x+N / 2} \eta_{h}\left(\tau_{i}^{x}\right)=\delta N$ then for firm $x k_{1}^{*}=k_{0}^{*}$ and so $\bar{f}_{0}=\bar{f}_{1}$.
} 
We now want to analyze the behavior of aggregate output over time. Let $Y_{t, t+1}$ be the aggregate output over the period $(t+1)-t$, e.g. the yearly GNP. As the number of firms at different sites is countable, we can define $Y_{t, t+1}=\sum_{x \in Z} Y_{x, t, t+1}$, where $Y_{x, t, t+1}$ is say the yearly output of the firm at site $x$. Of course this depends on the spillover effect from the neighbors and the technology used in that period. Suppose that firm $x$ capital stock becomes obsolete once in that period, say at $\tau_{2}^{x}$, $\tau_{1}^{x} \leq t \leq \tau_{2}^{x} \leq t+1$. Then

$$
Y_{x, t, t+1}=\int_{t}^{\tau_{2}^{x}} \bar{f}_{l} d t+\int_{\tau_{2}^{x}}^{t+1} \bar{f}_{m} d t
$$

for $l, m=0,1$. And so

$$
Y_{x, t, t+1}=\left(\tau_{2}^{x}-t\right) \bar{f}_{l}+\left((t+1)-\tau_{2}^{x}\right) \bar{f}_{m}
$$

Thus the aggregate output can show cyclical behavior because of two factors: (i) the randomness in the capital life at various sites, (ii) the changing of configurations of technology across sites. It should be noted that in this context the random times at which capital becomes obsolete at different sites play the role of exogenous shocks. As $\bar{f}_{0}<\bar{f}_{1},{ }^{5}$ hereafter a configuration where all firms use technology 1 will be called the "high equilibrium", while a configuration in which all firms use technology 0 will be called the "the low equilibrium".

\section{The Aggregate Process of Technological Choices: the Symmet- ric Case.}

We shall now analyze the aggregate process of technological choices. Each firm's productivity affects and is affected by the neighbors technology choices. Each firm has $N$ ( $N$ even) neighbors, $N / 2$ on the right and $N / 2$ on the left. For example the neighbors of firm $x$ are $x-N / 2, \ldots x-1, x+1, \ldots x+N / 2$. At each (random) time capital becomes obsolete the firm chooses the technology and the optimal stock of capital that ensure the maximization of its expected profits (1). Thus if at a random time $\tau_{i}^{x}, \sum_{h=x-N / 2, h \neq x}^{x+N / 2} \eta_{h}\left(\tau_{i}^{x}\right)>N / 2$ then firm $x$ chooses technology 1 , and if $\sum_{h=x-N / 2, h \neq x}^{x+N / 2} \eta_{h}\left(\tau_{i}^{x}\right)<$ $N / 2$ then firm $x$ chooses technology 0 . On the other hand whenever half of the neighbors are using 1 and half are using 0 then either technology gives the same expected profits, in this case we shall distinguish three different behavioral rules:

\footnotetext{
${ }^{5}$ Indeed when $\sum_{h=x-N / 2, h \neq x}^{x+N / 2} \eta_{h}\left(\tau_{i}^{x}\right)=\delta N$ then for firm $x \bar{f}_{0}=\bar{f}_{1}$.
} 
B1 the firm takes a draw from a uniform random variable on [0,1], if the realization is equal or smaller than $1 / 2$, it chooses technology 1 , otherwise it chooses technology 0.

B2 the firm switches technology.

B3 the firm keeps the technology currently used.

It is assumed that when half of the neighbors use 1 and half use 0 , then all firms follow either $\mathrm{B} 1$, or $\mathrm{B} 2$ or B3, that is we rule out the possibility that firms at different sites can follow different rules, or the possibility that a given firm chooses a different rule in different points in time. In the sequel we shall show that depending on the behavioral rule followed when half of the neighbors use 1 and half use 0 , the aggregate process of technological choices can be formally treated as either a linear or a non linear (threshold) voter model. Some further notation is now necessary:

$\eta_{x}^{L}(t)$ denotes the technology used by the firm at site $x$ at time $t$, for the case in which all firms follow rule B1. The corresponding aggregate process describing the (aggregate) configuration of technologies at time $t$ is denoted by $\eta^{L}(t)=\left(\eta_{x}^{L}(t), x \in Z\right)$, and we denote the aggregate processes of technologies $\eta^{L}=\left(\eta_{x}^{L}(t), x \in Z, t \geq 0\right)$. Similarly we denote $\eta_{x}^{c}(t), \eta^{c}(t)=\left(\eta_{x}^{c}(t), x \in Z\right)$, $\eta^{c}=\left(\eta_{x}^{c}(t), x \in Z, t \geq 0\right)$, and $\eta_{x}^{f}(t), \eta^{f}(t)=\left(\eta_{x}^{f}(t), x \in Z\right), \eta^{f}=\left(\eta_{x}^{f}(t), x \in Z, t \geq 0\right)$ the technology chosen by the firm at site $x$, the configuration of technology at time $t$ and the aggregate process of technology in the case all firms follow rule $B 2$ and $B 3$ respectively. Note that $\eta^{L}, \eta^{c}, \eta^{f}$ evolve on $\{0,1\}^{Z}$.

Hereafter let $|\cdot|$ denotes the Euclidean norm and \# denotes the cardinality of a set. In the sequel we shall use the following facts.

\section{FACT 3.1}

Let $\mathrm{A} 1$ and $\mathrm{A} 3$ hold, and all firms follow rule B1 and let $N=2$. Then,

$$
\eta^{L}=\left(\eta_{x}^{L}(t), x \in Z, t \geq 0\right)
$$

is a (nearest neighbor) linear voter model.

PROOF: $\eta^{L}$ is an interactive particle Markov process evolving on $\{0,1\}^{Z}$. Given $A 1$ and $A 3$, any time the firm can revise the technology choice, it looks at its two neighbors. Suppose that the firm was using technology 0, if no neighbor uses 1 (A4 rules out the possibility that at the same instant of time a neighbor can switch technology) then it keeps using technology 0 , on the other hand if both neighbors use technology 1 it switches to it, on the other hand if it was using technology 1 it 
switches if both neighbors are using 0 and does not switch if both neighbors use 1 . Thus the firm switches technology if both the neighbors disagree (on the technology choice), it does not switch if both agree and switches with probability $1 / 2$ if one agrees and the other does not. The value of the configuration $\eta^{L}$ at site $x$ changes at rate $c^{L}(x, \eta)$, where

$$
c^{L}(x, \eta)=\frac{1}{2} \#\left\{y:|y-x|=1, \eta_{x} \neq \eta_{y}\right\}
$$

Thus from Bramson and Griffeath (1980, p.183) we know that $\eta^{L}$ is a (nearest neighbor) linear voter model on a one-dimensional lattice ${ }^{6}$.

\section{FACT 3.2}

Let $\mathrm{A} 1$ and $\mathrm{A} 3$ hold, and all firms follow rule B2, with $N$ finite. Then

$$
\eta^{c}=\left(\eta_{x}^{c}(t), x \in Z, t \geq 0\right)
$$

is a threshold voter model with threshold equal to $N / 2$.

PROOF: $\eta^{c}$ is an interactive particle Markov process evolving on $\{0,1\}^{Z}$. Given A1 and A3 the firm switches if at least $N / 2$ neighbors are using a different technology. The value of the configuration $\eta^{c}$ at site $x$ flips at rate $c^{c}(x, \eta)$, where

$$
c^{c}(x, \eta)=1 \text { if } \#\left\{y:|y-x| \leq N / 2, \eta_{x} \neq \eta_{y}\right\} \geq N / 2 \text { and } 0 \text { otherwise }
$$

The statement then follows from Andjel, Liggett and Mountford (1992, p.74)

\section{FACT 3.3}

Let A1 and A3 hold, and all firms follow rule B3, with $N$ finite. Then

$$
\eta^{f}=\left(\eta_{x}^{f}(t), x \in Z, t \geq 0\right)
$$

is a threshold voter model with threshold equal to $N / 2+1$

PROOF: by the same argument used in the proof of Fact 3.2, by noting that

$$
c^{f}(x, \eta)=1 \text { if } \#\left\{y:|y-x| \leq N / 2, \eta_{x} \neq \eta_{y}\right\} \geq N / 2+1 \text { and } 0 \text { otherwise, }
$$

the statement follows from Durrett and Steiff (1993, p.232)

\footnotetext{
${ }^{6}$ The linear voter on the one-dimensional lattice is defined for the case in which each site just looks at the two neighboring sites.
} 
It should be noted that in the linear voter model, each site changes technology at rate proportional to the number of neighbors that disagree, while in the threshold voter the site changes technology if there is at least a given number (threshold) of neighbors that disagree.

Define $k^{l}=\left(k_{x}^{l}(t), x \in Z, t \geq 0\right), k^{c}=\left(k_{x}^{c}(t), x \in Z, t \geq 0\right), k^{f}=\left(k_{x}^{f}(t), x \in Z, t \geq 0\right)$ the aggregate process of capital stock when each firm follows rule B1, B2, B3 respectively. We have

\section{COROLLARY 3.4}

Let A1-A3 hold. Then the $k^{l}, k^{c}, k^{f}$ are respectively a linear voter (when we impose $N=2$ ), a threshold voter with threshold equal to $N / 2$ and to $N / 2+1$ respectively.

PROOF:

Suppose that at time $\tau_{i}^{x}$ the capital of firm $x$ becomes obsolete and for the $i$ - th time the firm has to reconsider the technological choice. Given that the firm is (expected) profit maximizer, if more than $N / 2$ neighbors use 1 it uses 1 , if less than $N / 2$ use 0 it uses 0 , and if exactly $N / 2$ use 1 it can choose either technology depending on which rule (B1,B2 or B3) it follows. Once the technology is chosen, it has to decide the optimal level of capital. We have seen the optimal stocks of capital for technology 1 and 0 respectively are $k_{1}^{*}$ and $k_{0}^{*}$. As switches in the optimal level of capital occur if and only if the technology switches, the statement follows.

Given the facts above, we can now analyze the dynamics and the asymptotic behavior of $\eta^{L}, \eta^{c}, \eta^{f}$, by borrowing some results already available in the probabilistic literature.

\section{The Dynamics and the Asymptotic Behavior of the Aggregate Technology Process}

As each firm will never change technology when all neighbors use the same technology, a configuration of either all firms using 1 or all firms using 0 is an absorbing state for the system. A first question that we wish to address is whether such configurations will be reached as $t$ gets large. More precisely we address the problem of whether consensus will eventually occur or not, where by consensus we mean that, as $t$ gets large, the probability that two generic sites (firms) use a different technology approaches zero. Thus consensus is a situation in which all sites will eventually agree on the same technology. We shall see below that consensus occurs when the flip rate are as in $(5,6)$, 
while consensus does not occur in general when the flip rates are as in (7).

Linear and nonlinear (threshold) voter models are non-ergodic, ${ }^{7}$ in the sense that the limiting distribution depends on the initial distribution. Several of the propositions stated below, require the initial distribution to be a Bernoulli product measure with density $\theta \in(0,1)$, denoted as $\mu^{\theta}$, that is $\mu^{\theta}\left(x: \eta_{x}(0)=1, x \in Z\right)=\theta, \theta \in(0,1)$. A4 ensures that the initial distribution is indeed a Bernoulli product measure. This means that at time 0 each site uses technology 1 with probability $\theta$ and technology 0 with probability $1-\theta$, independently of the choices of the other sites. We shall see that the voter process is nonergodic and a high value of $\theta$ increases the chances that a configuration of all ones will eventually occur. As in the case of all firms doing 1 , all positive spillovers are exploited and the aggregate production level is higher, consensus on technology 1 thus will be called the "high equilibrium". On the other hand, consensus on 0 implies that no positive spillover is exploited and the level of production is lower than it could be, and so it will be called the "low equilibrium" . Thus if the flip rates are as in $(5,6)$ either a high or low equilibrium will eventually occurs, with the former being more likely for higher value of $\theta$. On the other hand we shall see that if the flip rate is as in (7) the system in general will not converge to one of the two absorbing states and we shall have an infinite (but countable) number of mixed equilibria, in which some firms always use technology 1 and other will always use technology 0 . If the initial distribution is $\mu^{\theta}$, we start from a state of individual independence. The interaction between sites will then start at $t>0$, when sites (firms), at Poisson random times, will start reconsidering their technology choice by observing their neighbors.

\section{PROPOSITION 4.1}

Let A1,A3,A4 hold, then for any $x, y \in Z$,

$$
\text { (i) } \lim _{t \rightarrow \infty} \operatorname{Pr}\left(\eta_{x}^{c}(t)=\eta_{y}^{c}(t)\right)=1
$$

and

$$
\text { (ii) } \lim _{t \rightarrow \infty} \operatorname{Pr}\left(\eta_{x}^{L}(t)=\eta_{y}^{L}(t)\right)=1
$$

\footnotetext{
${ }^{7}$ This is in contrast with other interacting particle systems, such as the Ising models, that are instead ergodic. Ising models have been analyzed by Blume (1993) in order to study noisy best response dynamics.

${ }^{8}$ We shall see below that a long run configuration with all 0 implies the lowest possible level of aggregate activity achievable in equilibrium. Hence, we call it the low equilibrium. On the other hand, it is immediate to see that a long run equilibrium configuration of all 1 implies the highest possible level of aggregate activity. Hence, we call it the high equilibrium.
} 
PROOF: (i) Given A3,A4, from Fact 3.2 we know that $\eta^{c}$ is a threshold voter model with threshold equal to $N / 2$, A4 ensures that the initial distribution is translation invariant (i.e. it does not depend on the specific site), then the statement follows from Theorem 1a in Andjel, Liggett and Mountford (1992).

(ii) Given A3,A4, from Fact 3.2 we know that $\eta^{L}$ is a linear voter model with translation invariant initial distribution, the statement then follows from Cox and Griffeath (1986, p.350).

\section{COROLLARY 4.2}

Let A1-A4 hold and assume that whenever half of the neighbors use 1 and half use 0 , the firm follows either rule B1 or B2. Then the aggregate output will converge in probability to either to the high or to the low equilibrium.

PROOF: A2 ensures that the solution to the expected profit maximization is unique. As firms eventually agree on either technology 1 or 0 , either all firm exploit externalities (high output level) or none does (low output level)

Thus if $\forall x$ firm $x$ whenever $\sum_{h=x-N / 2, h \neq x}^{x+N / 2} \eta_{h}\left(\tau_{i}^{x}\right)=N / 2$ follows either the behavioral rule $\mathrm{B} 1$ or the behavioral rule B2 two only equilibria occur, the high equilibrium where each firm will eventually employ 1 or the low equilibria where each firm will eventually employ technology 0 . We shall show below that consensus implies cluster formation. For a cluster in $Z$ we mean a connected segment of sites using the same technology. For cluster size we mean the number of connected sites using the same technology. Hereafter the same argument applies to $\eta^{c}$ and to $\eta^{L}$, so for the sake of simplicity we drop the superscript. We can now define the mean cluster size around the origin at time $t$ as

$$
C\left(\eta^{\mu}(t)\right)=\lim _{n \rightarrow \infty} \frac{2 n}{\# \text { clusters of } \eta^{\mu}(t) \text { on }[-n, n]}
$$

where $\eta^{\mu}$ is either the linear or the threshold voter, with initial distribution $\mu$. We shall see below that, when consensus occurs, the mean cluster size grows with $t$ and approaches infinity as $t$ approaches infinity.

\section{PROPOSITION 4.3.}

Let A1,A3,A4 hold, then, as $t \rightarrow \infty, C\left(\eta^{c}(t)\right) \stackrel{p r}{\rightarrow} \infty\left(\operatorname{resp} . C\left(\eta^{L}(t)\right) \stackrel{p r}{\rightarrow} \infty\right)$.

PROOF: A4 ensures that the initial distribution is a Bernoulli product measure say $\mu_{\theta}$, A1 and A3 ensure that Fact 3.1 and 3.2 hold. As the proof is the same for both $\eta^{c}$ and for $\eta^{L}$ we shall drop 
the superscript $c, L$. We say that $x$ is a border for $\eta$ at time $t$, if $\eta_{x-1}(t) \neq \eta_{x}(t)$. A border measure for $\eta(t)$ is then defined as

$$
\mu^{\theta}\left(\eta(t): \eta_{x-1}(t) \neq \eta_{x}(t), x \in Z\right)
$$

The Bernoulli product measure with density $\theta \in(0,1)$ is translation invariant (i.e. it does not depend on the specific site) and it is also mixing (for a definition of mixing border measure, see Bramson and Griffeath 1980, p.187-188). Thus by the Birkoff ergodic theorem a strong law of large number follows, ensuring that with probability one,

$$
\lim _{n \rightarrow \infty} \frac{\# \text { of borders of } \eta(t) \text { in }[-n, n]}{2 n}=P(\eta(t) \text { has a border at } 1)
$$

As the number of borders and the number of clusters in $[-n, n]$ can differ by at most one, given the definition of mean cluster size, we have that

$$
C(\eta(t))=\frac{1}{P(\eta(t) \text { has a border at } 1)}=\frac{1}{P\left(\eta_{0}(t) \neq \eta_{1}(t)\right)}
$$

where the last equality follows straightforwardly from the definition of border at site 1 . Now from Proposition 4.1, we know that $\forall x, y \in Z$,

$$
\lim _{t \rightarrow \infty} \operatorname{Pr}\left(\eta_{x}(t)=\eta_{y}(t)\right)=1
$$

so that by setting $x=0, y=1$, we have that $\lim _{t \rightarrow \infty} \operatorname{Pr}\left(\eta_{0}(t) \neq \eta_{1}(t)\right)=0$, and the desired result then follows.

A more informative result is available for $\eta^{L}$, that is for the case in which when $\left.\sum_{h=x-N / 2, h \neq x}^{x+N / 2} \eta_{h}\left(\tau_{1}^{x}\right)\right)=$ $N / 2 \forall x \in Z$ firm $x$ follows behavioral rule B1 and $N=2$.

\section{PROPOSITION 4.4}

Under the same assumptions of Proposition 4.3, as $t$ gets large

$$
\frac{1}{\sqrt{t}} C\left(\eta^{L}(t)\right) \stackrel{p r}{\rightarrow} \frac{\sqrt{\pi}}{2 \theta(1-\theta)}
$$

PROOF: From Theorem 1c in Bramson and Griffeath (1980, p.191), since any Bernoulli product measure is mixing.

Proposition 4.4 shows that for the case of $\eta^{L}$, the size of clusters increases over time at rate $\sqrt{t}$. 
If A2 (ensuring a unique solution to the expected profit maximization) holds too, then whenever firms follows either rule B1 or B2, along the dynamics we shall observe over time larger and larger areas of firms using either technology 1 (wealthy areas) or technology 0 (poor areas). As the cluster size (at least for the linear voter) grows at a rate slower than $t$, the clusters seem to be stationary across the dynamics. This is consistent with the stylized fact that we observe large wealthy areas, alternated by large poor areas.

We have stated Propositions 4.1, 4.3 and 4.4 in terms of a countable number of firms. It is worthwhile to see whether the same results hold for the case of a finite population. Basically this is true for Proposition 4.1, in fact Part (ii) follows from Cox (1989, p.1333), and Part (i) follows from the fact that no configurations, but all 1 and all 0 , regardless of the number of agents, can be stable over time. On the other hand, Propositions 4.3 and 4.4 hold only for the countable case. An intuitive reason is that, over a segment of finite length $n$, we cannot have a cluster growing over time, as time approaches infinity. On a more formal level, we notice that in the proof of Proposition 4.3 we make use of the Birkoff ergodic theorem, that holds only for $n \rightarrow \infty$.

Thus for $\eta^{c}$ and $\eta^{L}$, as $t$ gets large we get consensus on only one technology, that is either the high or the low equilibrium will eventually occur. Furthermore, as $\eta^{c}, \eta^{L}$ are non-ergodic processes, their limiting distribution will depend on the initial distribution. In particular, as we shall see below, the limiting distribution is a mixture of pointmass distributions $\mu^{1}, \mu^{0}$, where $\mu^{1}\left(r e s p . \mu^{0}\right)$ denotes a configuration in which all firms use 1 (resp. 0). The weights of this mixture depend on the initial state of the system. Hereafter with $\mu^{c}(t), \mu^{L}(t)$ we denote the distribution of the configuration $\eta^{c}(t), \eta^{L}(t)$, respectively, at time $t$. We have

\section{PROPOSITION 4.5}

Let A1,A3,A4 hold, then

$$
\begin{gathered}
\text { (i) } \lim _{t \rightarrow \infty} \mu^{L}(t)=\theta \mu^{1}+(1-\theta) \mu^{0} \\
\text { (ii) } \lim _{t \rightarrow \infty} \mu^{c}(t)=D(\theta) \mu^{1}+(1-D(\theta)) \mu^{0}
\end{gathered}
$$

for some constant, depending on $\theta, 0<D(\theta)<1$ and increasing in $\theta$.

PROOF: (i) It is shown in Ianni and Corradi (2000, Theorem 2.2) that the assumptions of Corollary 1.13(a) of Liggett (1985, p.231) are satisfied. The statement then follows from that Corollary.

(ii) From Theorem 1b in Andjel, Liggett and Mountford (1992). 
Thus from Proposition 4.5 we know that with probability $\theta,($ resp. $D(\theta)), \mu^{L}(t),\left(\right.$ resp. $\left.\mu^{c}(t)\right)$ converges to a configuration of all using 1 , and with probability $1-\theta,($ resp. $1-D(\theta)), \mu^{L},\left(\right.$ resp. $\left.\mu^{c}\right)$ converges to a configuration of using 0 . So the only difference in the limiting distribution of $\mu^{L}(t)$ and $\mu^{c}(t)$ lies in the weights of the mixture of pointmass distributions.

We have seen in Corollary 4.2 that given A1-A4, if firms follow either rule B1 or rule B2 then we have two equilibria, the low and the high equilibrium, given Proposition 4.5 we can see that the high equilibrium is more likely for larger values of $\theta$.

If whenever half of the neighbors use 0 and half use 1 the firm keeps the technology currently used (rule B $3^{9}$ ), then in general we do not have consensus on only one technology, and so we do not observe cluster formation along the dynamics. Intuitively the main difference between the dynamic behavior of $\eta^{f}(t)$ and that of $\eta^{L}(t), \eta^{c}(t)$ is the following: as a firm switches technology only if more than half of her/his neighbors disagree (i.e. if at least $N+1$ neighbors disagree), any time the site switches, the number of sites in her/his neighborhood that disagree on the technology held by that site, decreases (by one). For this reason we expect that over time, each site switches less frequently than in the other previous two cases. In fact, as we shall see below, any firm can switch technology only a finite number of times. Furthermore intuition suggests that both the technologies will in general survive in the long-run, so that we may have co-existence. For example if $N=2$, all configurations characterized by two or more connected sites using 1 , followed by two or more connected sites using 0 , and so on, are stable over time and can be seen as stationary limiting configurations. In fact we shall have an entire family of stationary configurations, characterized by the co-existence of technologies. Nevertheless also a configuration of all 1 and a configuration of all 0 are stationary limiting distributions. Thus in this case we shall have also an entire family of equilibrium aggregate output. Furthermore two (different) configurations characterized by the same probability measure over site employing technology 1 and sites employing 0 gives rise to different aggregate output, depending on how connected are the sites using the same technology. Thus in this case we have an infinite (but countable) number of long-run aggregate output. As configurations with less than $N / 2$ connected strings of either 0 or 1 cannot be stable, we see that the lowest level of aggregate economic activity occurs in the case of a limiting configuration with all 0 (that is no negative externalities from neighbors can survive in the long run). We shall see

\footnotetext{
${ }^{9}$ This rule seems to be the more realistic, in that it captures the unwilligness of a firm to switch technology when the returns from either technology are the same.
} 
below that, when the number of neighbors is finite, but large, configurations characterized by large relatively homogeneous areas are likely to be observed. We have

\section{PROPOSITION 4.6}

Let $\mathrm{A} 1, \mathrm{~A} 3, \mathrm{~A} 4$ hold. If whenever half of the neighbors use 0 and half use 1 the firm follows rule B3, then each firm switches technology only a finite number of times and the system fixates, that is $\forall x \in Z$, with probability one

$$
\lim _{t \rightarrow \infty} \eta_{x}^{f}(t)=\eta_{x}^{f}(\infty)
$$

PROOF: Let $\delta_{x, y}(t)=1$ if $\eta_{x}^{f}(t) \neq \eta_{y}^{f}(t)$, and 0 otherwise. Along the lines of Durrett and Steiff (1993, Theorem 1), we can define an energy function at time $t$, as

$$
\Upsilon_{t}=\sum_{x, y:|x-y| \leq N / 2} e^{-|x+y|} \delta_{x, y}(t)
$$

with $\Upsilon_{0}<\infty$. Let $\Upsilon_{t}(x)$ be the energy at site $x$, i.e.

$$
\Upsilon_{t}(x)=\sum_{y:|x-y| \leq N / 2} e^{-|x+y|} \delta_{x, y}(t)
$$

We shall show that $\forall x \in Z$, whenever the site flips, the energy at that site drops by a strictly positive amount. For the sake of simplicity, take $x=0$. Thus we have:

$$
\Upsilon_{t}(0)=\sum_{y=-N / 2}^{N / 2} e^{-|y|} \delta_{0, y}(t)
$$

Note that whenever $\sum_{y=-N}^{N} \delta_{0, y}(t)<N / 2$ site 0 will not flip anymore, and when this occurs for all sites in $Z$ the system fixates. Now let $\alpha=\#\left\{y \in\{-N / 2, \ldots,-1,0,1, \ldots N / 2\}: \eta_{0}^{f}(t) \neq \eta_{y}^{f}(t)\right\}$ (i.e. $\alpha$ is the number of sites disagreeing with the origin at time $t$ ). Suppose that at time $t$ site 0 flips, and recall that this can only happen if $\alpha>N / 2$. Then the drop in the energy at site 0 , after the flip occurred, is bounded from below by

$$
e^{-N}(\alpha-(N-\alpha))
$$

which is strictly positive as (i) $N / 2$ is a finite integer (ii) $\alpha>N / 2$. Thus we have a strictly positive drop of energy at site 0 . The same argument applies to any generic site. Thus as any site drops energy whenever it flips, and the energy at time 0 is finite, it follows that each site can flip only a finite number of times, so that fixation occurs. 
Thus when the flip rate is as in (7), as $t$ gets large, both technologies may survive and can coexist as time gets large. In particular we have infinitely many stationary configurations $\eta^{f}(\infty)$; as we mentioned above for $N=2$, configurations as . . 11001100110011 . . or . . 1111000111000111 . . are stationary. ${ }^{10}$ For $N$ finite but sufficiently large, even if, in general, we may not have consensus (and hence we may not have clustering), we shall tend to observe large regions characterized by the prevalence of one technology alternated by large regions characterized by the prevalence of the other technology. In fact any configuration characterized by segments of less than $N$ consecutive sites using the same technology, cannot be stable over time, and so cannot be a stationary limiting configuration.

\section{COROLLARY 4.7}

Let A1-A4 hold. If whenever half of the neighbors use 0 and half use 1 the firm follows rule B3, then we shall have infinitely many long run equilibria for the aggregate output.

Finally it is worthwhile to point out that Proposition 4.6 (and hence Corollary 4.7) holds also for the case of a finite population. In fact the proof of that proposition is valid, regardless the number of sites we consider. In particular, as each site can switch technology only a finite number of times, if we have finitely many sites, fixation will occur in finite time.

So far we have considered the case of $\delta=1 / 2$ and of symmetric flip rate, in the sense that the flip rate from 0 to 1 is the same as that from 1 to 0 . In the next section we shall consider the case of asymmetric rate, i.e. of $\delta \neq 1 / 2 .{ }^{11}$ It is worthwhile to analyze the relationship between the value of the threshold parameter $\delta$ and the strength of technological complementarities. Durlauf (1993) measures the strength of technological complementarities in terms of the probability that a firm use technology 1 when some (possibly all) of its neighbors use 0 . The higher is this probability, the weaker is the degree of technological complementarities. Using Durlauf notation (eq.3.2, 1993), the strongest spillover effect occur when $\theta_{\min }=\theta_{\max }=1$, in terms of our model, this means that a firm chooses technology 1 even if all neighbors are using 0 , that is $\delta=0$. Thus we shall call strong (weak) strategic complementarities the case of $\delta<\frac{1}{2}(\delta>1 / 2)$ and we shall call intermediate strategic complementarities the case of $\delta=1 / 2$. From the results stated above, we see that in the case of $\delta=1 / 2$ and symmetric flip rate we shall have either two or infinitely many equilibria, provided we

\footnotetext{
${ }^{10} \mathrm{No}$ configuration with a 1 surrounded by two 0 (or viceversa) can survive in the long run.

${ }^{11}$ The case in which $\delta=1 / 2$ but whenever half of the neighors are using 1 the sites always uses 1 (or 0 ) also falls in the class of asymmetric flip rates.
} 
start from a translation invariant initial distribution. Thus in our model, growth is nonergodic in the case of spillovers of intermediate strength.

\section{The Aggregate Process of the Technologies: The Asymmetric Case}

At each (random) time capital becomes obsolete the firm chooses the technology and the optimal stock of capital that ensure the maximization of its expected profits (1). Thus if at a random time $\tau_{i}^{x}, \sum_{h=j-N / 2, h \neq x}^{x+N / 2} \eta_{h}\left(\tau_{i}^{x}\right)>\delta N$ then firm $x$ chooses technology 1 , and if $\left.\sum_{h=x-N / 2, h \neq x}^{x+N / 2} \eta_{h}\left(\tau_{i}^{x}\right)\right)<$ $\delta N$ then firm $x$ chooses technology 0 , and this holds for all $x \in Z$. On the other hand whenever a fraction $\delta$ of neighbors use technology 1 and a fraction $(1-\delta)$ use 0 , then either technology gives the same expected profits. In this case we shall distinguish three different behavioral rules, $\forall x$ :

C1 the firm chooses technology 1

C2 the firm chooses technology 0

C3 the firm takes a draw from a uniform random variable on $[0,1]$, if the realization is equal or smaller than $1 / 2$ chooses technology 1 , otherwise it chooses technology $0 .{ }^{12}$

It is easy to notice that, whenever $\delta<1 / 2$, the flip rates generated by the above rules are asymmetric, in the sense that to flip from 0 to 1 it suffices to have $N \delta$ (or $N \delta+1$, depending on the rule) neighbors disagreeing, while to flip from 1 to 0 it requires $N(1-\delta)$ (or $N(1-\delta)-1$ depending on the rule). Similarly if $\delta=1 / 2$ and each firms follows rule $\mathrm{C} 1$ then it requires $N / 2$ neighbors doing 1 to flip from 0 to 1 , while it requires $N / 2+1$ neighbors doing 0 to flip from 1 to 0 . Let us define $\eta^{C 1}(t), \eta^{C 2}(t), \eta^{C 3}(t)$, the processes defined by rule $\mathrm{C} 1, \mathrm{C} 2$ and $\mathrm{C} 3$ respectively.

\section{PROPOSITION 5.1}

Let A1, A3 hold and suppose that firms follow rule (i) and $\delta \in(0,1 / 2]$.

Define $E_{t}$ the following event:

$E_{t}=($ at time $t$ there is a string of at least $\delta N+1$ consecutive sites using 1$)$. Also define

$$
\tau_{E}=\left\{\inf t: E_{t} \text { occurs }\right\}
$$

\footnotetext{
${ }^{12}$ Note that these behavioral rules do not correspond with those stated in the symmetric case, i.e. $\delta=1 / 2$.
} 
If $P\left(\tau_{E}<\infty\right)=1$, then

$$
\lim _{t \rightarrow \infty} \operatorname{Pr}\left(\eta_{x}^{C 1}(t)=1, \forall x \in Z\right)=1
$$

PROOF:

For the sake of simplicity, and without loss of generality, assume that $\delta=1 / 2, N=6$, so that $\delta N+1=4$, thus a firm using 0 switches to 1 if at least two neighbors are using 1 . Consider the most unfavorable situation (in terms of prevalence of firms using 1), in which $E_{t}$ occurs at some finite $t$. This is

$$
\text { ..00000000111100000000... }
$$

At any (random) time a firm using 1 has a chance to revise its technology, it does not flip; the same holds for all firms using 0 , but for the two on the immediate left and right of the string of 1 . When any of them has a chance to revise its technology, it will switch to 1 . As in any period firm site has a positive probability of revising its technology, we shall next have a configuration

$$
\text { ..0000001111111000000 .. }
$$

by the same argument above when any of the two 0-sites on the immediate left and right of the cluster of 1 will be called to revise its technology it will switch to 1 , while no site will ever switch to 0 . Thus as $t \rightarrow \infty$ we shall have consensus on 1 . Note that a similar argument holds for any arbitrary $\delta<1 / 2$.

We shall now see that A4 ensures that the statement of Proposition 5.1 necessarily holds.

\section{PROPOSITION 5.2}

Let A1,A3,A4 holds. Then

$$
\lim _{t \rightarrow \infty} \operatorname{Pr}\left(\eta_{x}^{C 1}(t)=1, \forall x \in Z\right)=1
$$

\section{PROOF:}

A1 ensures that at time 0 each sites employs technology 1 with probability $\theta$ and technology 0 with probability $(1-\theta)$. It suffices to show that the event $E_{t}$, occurs at $t=0$, with probability one, then the statement follows from Proposition 5.1. We shall consider the case of M firms, lying on $-M / 2,-M / 2+1, \ldots-1,0,1,2, \ldots M / 2$ and then let $M$ go to infinity. From a very large urn with a proportion $\theta$ of 1 -balls (i.e. firms using 1 ) and $1-\theta$ of 0 -balls we draw with replacement 
$(M /(N \delta+1)) N \delta+1$-tuple of balls (e.g. if $\delta=1 / 3$, and $N=6$ we draw 3 balls at once, put them back in to the urn, and repeat the process $M / 3$ times). As the urn is very large, if we draw $N \delta+1$ balls consecutively, the chance that the first is say 1 is the same that the second or the third is 1 . We proceed as follows: we first draw $N \delta+1$ balls and place them on sites $-M / 2,(-M / 2)+1, \ldots(-M / 2)+N \delta+1$, put the $N \delta+1$ balls back in to the urn, pick another $N \delta+1$-tuple, and place them on sites $(-M / 2)+N \delta+2, \ldots(-M / 2)+2(N \delta+1)$, and so on until we have covered all the $M$ sites. The probability of getting a $N \delta+1$-tuple of 1 s is given by $\theta^{N \delta+1}$, while $1-\theta^{N \delta+1}$ is the probability that at least a ball in the $N \delta+1$-tuple is a 0 . Hence

$$
\operatorname{Pr}\left(E_{0}\right)=1-\left(1-\theta^{N \delta+1}\right)^{M /(N \delta+1)} \rightarrow 1 \text { as } M \rightarrow \infty
$$

The desired result then follows from Proposition 5.1.

It is immediate to see that Proposition 5.1 (as well as Proposition 5.3 below) holds also for a finite population, while this is not the case for Proposition 5.2.

Thus if at any given finite time we have a string of at least $N \delta+1$ consecutive sites using $A$, we shall have consensus on 1 , as $t \rightarrow \infty$. This property of the asymmetric threshold voter is related to the concept of contagion proposed by Morris (2000), for the case of coordination games. For contagion is meant a situation in which whenever the risk dominant strategy is played by a minimum number of neighboring players, it will eventually spread out to all the population. In Ellison (1993), Blume (1995) and Morris (2000), the contagion threshold in 1/2 on a one-dimensional lattice, but it is strictly smaller than $1 / 2$ on a two dimensional lattice. Lee and Valentinyi (2000) improve upon this, by showing that, provided the initial distribution is random, the contagion threshold is $1 / 2$ also on a two-dimensional lattice. The main difference between the set up considered in the papers cited above and our set up, in that in the former all players (sites) have the possibility of revising their strategy simultaneously at discrete time interval, while in the latter at any instant of time any site have a positive probability of revising its strategy (technology), but the probability that two sites revise their technology simultaneously is zero. For this reason we have a contagion threshold equal to $1 / 2$ on a one dimensional lattice ${ }^{13}$, but we would have a threshold of $1 / 4$ on a two dimensional lattice. ${ }^{14}$

We now consider the two nonlinear rules $\mathrm{C} 2$ and $\mathrm{C} 3$.

\footnotetext{
${ }^{13}$ This occurs when $\delta=1 / 2$ and all firms follow rule C1.

${ }^{14}$ This can be easily down using the same argument as in the proof of Propositions 5.1 and 5.2. In fact we could
} 


\section{PROPOSITION 5.3}

Suppose that each firms follows rule (C2) (resp. (C3)) and $\delta \in(0,1 / 2)$.

Define $E_{t}$ the following event:

$E_{t}=$ (at time t there is a string of at least $N \delta+2$ consecutive sites favoring A). Also define

$$
\tau_{E}=\left\{\inf t: E_{t} \text { occurs }\right\}
$$

If $P\left(\tau_{E}<\infty\right)=1$, then

$$
\begin{gathered}
\lim _{t \rightarrow \infty} \operatorname{Pr}\left(\eta_{x}^{C 2}(t)=1, \forall x \in Z\right)=1 \\
\text { (resp. } \left.\lim _{t \rightarrow \infty} \operatorname{Pr}\left(\eta_{x}^{C 3}(t)=1, \forall x \in Z\right)=1\right)
\end{gathered}
$$

PROOF:

The proof is the same for both rules. Without loss of generality set $\delta=1 / 3, N=6$, so that $N \delta+2=4$. Suppose the most unfavorable situations for 1 in which $E_{t}$ may occur, i.e.

$$
\ldots 00000000111100000000 \ldots
$$

Note that under both rule $\mathrm{C} 2$ and $\mathrm{C} 3$, no site using 1 will flip to 0 when has a chance to revise its technology. Now in both cases the two site 0-sites on the immediate left and right of the 1-string, would flip to 1 , while the second two immediate 0 on the left and right of the 1 group will not flip in case $\mathrm{C} 2$ and would flip to 1 with probability $1 / 2$, in case $\mathrm{C} 3$. The result then follows from the same argument given in the proof of Proposition 5.1.

\section{COROLLARY 5.4}

Let A1-A4 hold. If either of the following holds (i) each firm follows rule $\mathrm{C} 1$ and $\delta \in(0,1 / 2]$, (ii) each firm follows rule $\mathrm{C} 2$ (or $\mathrm{C} 3$ ) and $\delta \in(0,1 / 2)$, then we have a unique "high" long run equilibrium aggregate output, where all positive externalities are exploited, regardless the initial distribution. If instead either of the following hold (i) each firm follows rule $\mathrm{C} 2$ and $\delta \in[1 / 2,1$ ) (ii) each firms follows rule $\mathrm{C} 1$ (or $\mathrm{C} 3$ ) and $\delta \in(1 / 2,1)$, then we have a unique "low" long run equilibrium aggregate output, where no positive externality is exploited, regardless the initial distribution.

show that whenever we have a square with side larger or equal than $N \delta+1, \delta \leq 1 / 4$ and firms follow rule $\mathrm{C} 1$, then we shall have convergence on a configuration of all 1. 


\section{Conclusions}

In this paper we have proposed a simple model of local interaction between firms producing an homogeneous goods. Capital becomes obsolete at random Poisson time, and has an average life equal to one. Whenever capital becomes obsolete, the firm can reconsider its technology choice. A profit maximizing firm choose technology 1 when the number of neighbors using that technology is above a given threshold, and otherwise choose technology 0 . We have multiple long run equilibria, and so nonergodic growth, when the flip rate symmetric. This can occur under two circumstances: (i) the probability of switching from technology 1 to technology 0 and from technology 0 to technology 1 is the same, (ii) to switch from 0 to 1 (or from 1 to 0 ) it requires the same number of neighbors using technology 1 (0). Also, depending on the behavioral rule followed by the firm when half of the neighbors use technology 1 and half use technology 0 , we have either two equilibria or infinitely many equilibria. We have a unique long run equilibrium when the flip rates are asymmetric. More precisely, if it requires a smaller number of neighbors using technology 1 to switch from 0 to 1 , then we have convergence to a high equilibrium, with all firm using technology 1 . This is consistent with the recent game theoretical literature showing that if the threshold parameter if low enough, then we shall have convergence with probability one to the risk dominant strategy. On the other hand, our results differ from Durlauf's (1993); in fact he shows that when technological complementarities are weak we have convergence to the high equilibrium, while when technological complementarities are strong we have multiple equilibria. This is mainly due to the different modeling of technological spillover. Hence our conclusion is that while technological spillovers are a possible explanation of nonergodic growth, the fact that either weak, or strong or intermediate technological complementarities leads to nonergodic (or ergodic) growth depend on the specific modeling of the spillover transmission. 


\section{$7 \quad$ References}

Andjel E.D., T.M. Liggett and T. Mountford (1992), Clustering in one-dimensional threshold voter models, Stochastic Processes and their Applications , 42, 73-90.

Azariadis C. and A. Drazen (1990), Threshold externalities in economic development, Quarterly Journal of Economics, 105, 501-526.

Bala V. and G. Sorger (1999), A spatial-temporal model of human capital accumulation, Journal of Economic Theory, forthcoming.

Blume L. E. (1993), The statistical mechanics of strategic interaction, Games and Economic Behavior, 5, 387-424.

Blume L.E. (1995), The statistical mechanics of best-response strategy revision, Games and Economic Behavior, 11, 111-145.

Bramson M. and D. Griffeath (1980), Clustering and dispersion rates for some interactive particle systems on Z, Annals of Probability, 7, 418-432.

Brock W.A. and S.N. Durlauf (2000), Interactions-based models, forthcoming in Handbook of Econometrics, v.5, Heckman J.J. and E. Leamer (eds.), North-Holland Elsevier Science.

Cooper R. and A. John (1988), Coordinating failure in keynesian models, Quarterly Journal of Economics, 103, 441-465.

Cox J.T. and D. Griffeath (1986), Diffusive clustering in the two dimensional voter model, Annals of Probability, 14, 347-370.

Cox J.T. (1989), Coalescing random walks and voter model consensus times on the torus in $Z^{d}$, Annals of Probability, 17, 1333-1366.

Durlauf S.N. (1993), Nonergodic growth, Review of Economic Studies , 60, 349-366

Durlauf S.N. (1994), Spillovers, stratification and inequality, European Economic Review, 38, $836-845$.

Durlauf S.N. and D.T. Quah (1999), The new empirics of economics in growth, forthcoming in Handbook of Macroeconomics, Ch.4, 231-304, Taylor J.B. and M. Woodford (eds), North-Holland Elsevier Science.

Durrett R. and J.E. Steiff (1993), Fixation results for threshold voter, Annals of Probability, $21,232-247$ 
Ellison G. (1993), Learning, local interaction, and coordination, Econometrica, 61, 1047-1071

Ianni A. and V. Corradi (2000), Consensus, contagion and clustering in a space-time model of public opinion formation, Mimeo, University of Southampton

Lee I.H. and A. Valentinyi (2000), Noisy contagion without mutation, Review of Economic Studies, v.67, 47-56.

Liggett T.M. (1985), Interacting Particle Systems, Springer and Verlag, Berlin

Möbius M. (1999), The formation of ghettos as a local interaction phenomenon, Mimeo, MIT.

Morris S. (2000), Contagion, Review of Economic Studies, v.67, 57-78.

Murphy K., A. Shleifer and R. Vishny (1989) Industrialization and the big push, Journal of Political Economy, 97, 1003-1026.

Solow R. (1956) A contribution to the theory of economic growth, Quarterly Journal of Economics, LXX, 65-94. 